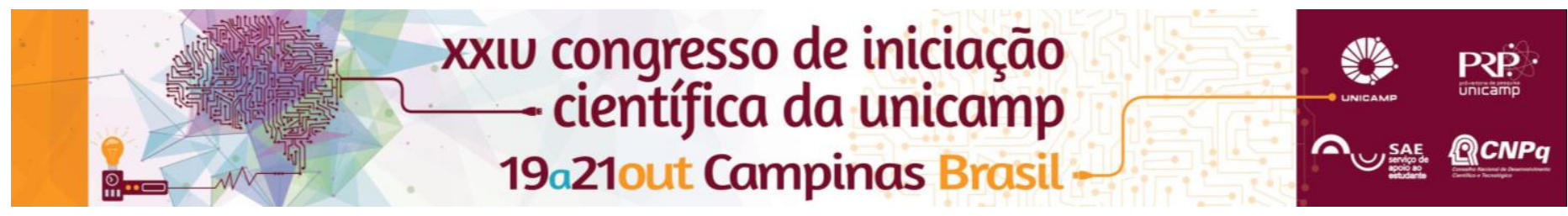

\title{
Multimodal evaluation of grey and white matter in patients with generalized epilepsy according to seizure control
}

\author{
Marina S. Polydoro*, Danielle S. Garcia, Marina Alvim, Fernando Cendes, Clarissa L. Yasuda
}

\begin{abstract}
Epilepsy is a neurological condition that affects approximately 50 million people. Usually, the magnetic resonance imaging (MRI) in idiopathic generalized epilepsy (IGE) patients is normal. However, several studies found conflicting results about subtle abnormalities in brain structures and cortical function of these patients after MRI quantitative and functional evaluations. The majority of them do not emphasize the relationship between seizure control by medication and the MRI results. The purpose of this project is to clinically characterize and evaluate both white and gray matter abnormalities in IGE patients according to their treatment response. The clinical response of 33 IGE patients was analyzed based on their first and last appointments at Hospital das Clinicas/Unicamp's Epilepsy service. The prevalence of seizure had a statistic significant decreased from the first to the last year of following, as the prevalence of generalized tonic-clonic seizure in this period. However, frequencies of absence seizure and myoclonic seizure remained. More than half of the patients presented at least one seizure in the last year of following. The results of imaging analysis showed statistic significant reduction of subcortical gray matter volume of the patient group in comparison with the control group; subgroup analyses revealed larger volumes in the refractory group. The clinical results shows that despite the significant decreased of prevalence of seizures, IGE epilepsy it is not easily control in the majority of times, and the type of seizure more difficult to control is the myoclonic seizure. As for the imaging analyses, the results were unexpected because the hypothesis was that if the thalamic volume is reduced in the patients in general, the refractory patients would have a more important reduction.
\end{abstract}

Key words: IGE patients, MRI, Treatment response

\section{Introduction}

Epilepsy is a neurological condition that affects approximately 50 million people ${ }^{1}$. Generalized epilepsy results in seizures involving the whole cerebral cortex ${ }^{2}$, one of the subtypes is the idiopathic generalized epilepsy (IGE). Usually, the magnetic resonance imaging (MRI) in IGE patients is normal. However, several studies found subtle abnormalities in brain structures and corticsl function assessed with quantitative and functional MRI evaluations ${ }^{3}$. Nevertheless, these studies also identified conflicting results. The majority of them do not emphasize the relation between seizure control by medication and the MRI results. The purpose of this project is to clinically characterize and evaluate the white and gray matter abnormalities in IGE patients according to their treatment response.

\section{Results and Discussion}

The clinical evaluation was conducted through the patients medical records analysis, and white and gray matter changes will be assessed by $3 \mathrm{~T} \mathrm{MRI}$ and postprocessing with VBM/Dartel, diffusion tensor image analysis and FreeSurfer analysis.

The clinical response of 33 IGE patients was analyzed based on their first and last appointments at Hospital das Clinicas/Unicamp's Epilepsy service. The prevalence of seizure presented a statistic significant decreased from the first to the last year of following $(p=0.004)$. According to different types of seizures, the generalized tonic-clonic seizure presented significant decrease in this period $(p=0.02)$, while the prevalence of absence seizure presented a non-significant decrease in this period $(p=0.17)$. Finally, there wasn't significant difference in the prevalence of myoclonic seizures in this period. More than half of the patients presented at least one seizure in the last year of following. These results shows that despite the significant decrease of prevalence of seizures, IGE epilepsy it is not easily control in the majority of times, and the most difficult type to control is the myoclonic seizure.

As for the imaging analysis, the MRI of 158 subjects were analysed. The control group consisted in 40 subjects and the patient group was composed by 21 patients controlled by treatment and 19 patients refractory to treatment. The results showed significant reduction of subcortical gray matter volume $(p<0.05)$ of the patient group in comparison with control group. The thalamic volume of the controlled patients was reduced in comparison with the refractory patients $(p<0.05)$. This final result was surprising as it was expected a more severe reduction in refratory group.

\section{Conclusions}

These results show that despite common knowledge in literature, IGE isn't an easy control kind of epilepsy.

\section{Acknowledgement}

Sponsors Institutions: CNPq from August/15 to October/15; FAPESP from November/15 to October/16.

\footnotetext{
${ }^{1}$ Browne T.R., Holmes G.L. Epilepsy. $N$ Engl J Med. 2001. 344(15): 1145 1151

Berg, A.T., et al.. Revised terminology and concepts for organization of seizures and epilepsies: report of the ILAE Commission on Classification and Terminology, 2005-2009. Epilepsia. 2010, 51, 675-85.

Betting L.E., et al. MRI volumetry shows increased anterior thalamic volumes in patients with absence seizures. Epilepsy Behav. 2010, 8, 575-80.
} 